\title{
Secondary Late Epidural Abscess After Deep Cervical Infection
}

\author{
P Kara, O Yavasi, G Kirimli, E Unluer, C Yilmaz
}

\begin{abstract}
Citation
P Kara, O Yavasi, G Kirimli, E Unluer, C Yilmaz. Secondary Late Epidural Abscess After Deep Cervical Infection. The Internet Journal of Emergency Medicine. 2009 Volume 6 Number 2.
\end{abstract}

DOI: $\underline{10.5580 / 21 \mathrm{~d} 0}$

\begin{abstract}
Abscess formation along the spinal epidural space is a rare condition. Predisposing factors include spinal surgery, recent trauma, immunosuprression, a distal site of infection and intravenous drug use. The classical clinical triad, known as fever, spinal pain and neurological deficit, is observed in only a small portion of the cases. These classical symptoms are non-specific and regrettably in crowded EDs. It is hard to diagnose spinal epidural abscess early because of the nonspecific nature of its symptoms. The lack of related sensitive and specific laboratory tests adds further to the difficulty of early diagnosis. We describe a patient with no significant predisposing factors other than previous history of deep neck infection that occured two months ago who presented with particularly back pain.
\end{abstract}

\section{INTRODUCTION}

Abscess formation along the spinal epidural space is a rare condition with incidence rates of $0.2-2.8$ per 10.000 cases in population per year. ${ }^{1}$ Predisposing factors include spinal surgery, recent trauma, immunosuprression, a distal site of infection and intravenous drug use. ${ }^{2}$ The typical symptoms of back pain and fever are common daily presenting complaints in the emergency department (ED); therefore the condition is easily missed if physician fail to consider it in the differential diagnosis. Multipl ED visits and diagnostic delays ocur in $68 \%$ of patients, contributing to a $45 \%$ morbidity rate and $15 \%$ mortality. We describe a patient with no significant predisposing factors other than previous history of deep neck infection that occured two months ago who presented with particularly back pain.

\section{CASE REPORT}

A 55-year-old female presented to the emergency department in March 2010, complaining of back pain and numbness in her lower extremities for the last week. Her past medical history was unremarkable except a deep cervical staphylococcal infection two months ago which was treated with antibiotic therapy and surgical drainage in our institute. CT revealed an anterior neck abcess (Fig 1). She denied any recent trauma. Her vital signs were unremerkable. Physical examination revealed hyperactive deep tendon reflexes in the lower extremities. Motor power examination revealed a $1 / 5$ score for both lower extremities. Abnormal laboratory tests were: white bllod cell count (WBC) of $11.3 \times 10^{9}(4.5-11) \mathrm{K} / \mathrm{uL}$, platelet 435 (130-400) $\mathrm{K} / \mathrm{uL}$, erythrocyte sedimentation rate (ESR) of 118 (0-30) $\mathrm{mm} / \mathrm{h}$ and a C-reactive protein (CRP) of $9.46(0.01-0.82)$ $\mathrm{mg} / \mathrm{dl}$. All the other laboratory tests (liver function enzyms, renal function parameters, coagulation profile) were within normal limits. 


\section{Figure 1}

Fig. 1 A transverse CT image of the neck reveals an anterior soft tissue mass with ill-defined margins. Also noted are gas pockets within the mass indicative of an abscess (arrow).

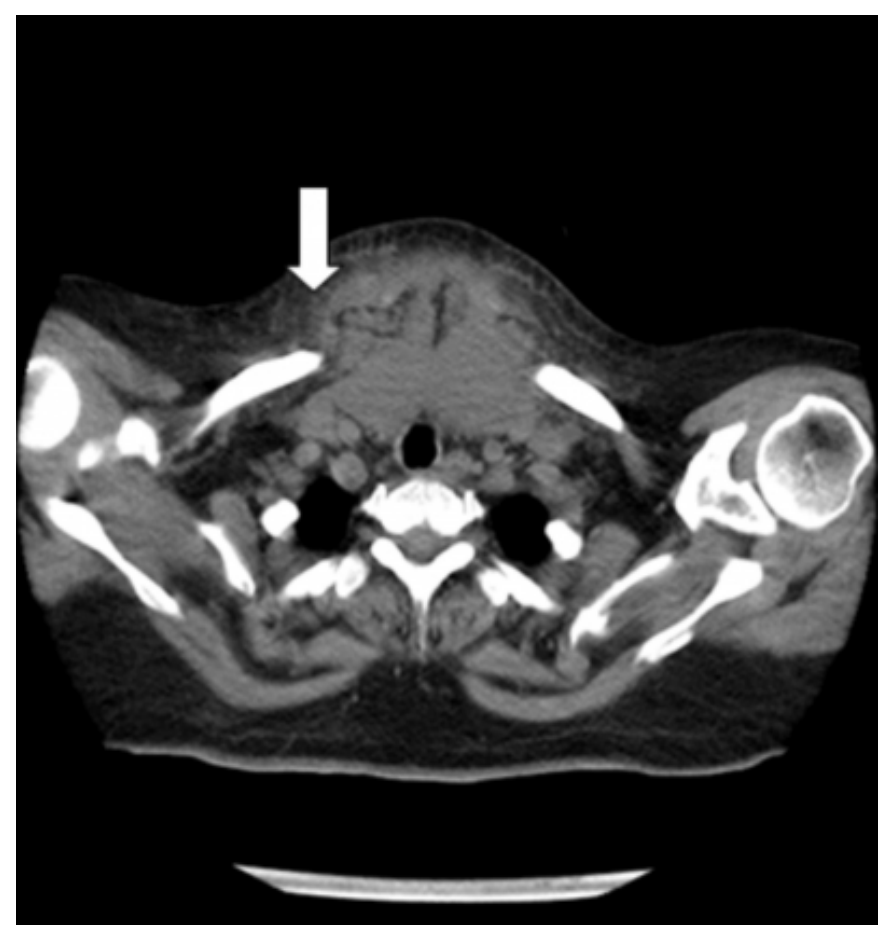

Directly an MRI was ordered including the thoracic and lumbar region. MRI demonstrated changes consistent with spondylodiscitis of thoracic vertebrae and enhanced MR image at the same level revealed a mass in the T 8-10 posterior epidural space compressing the spinal cord and causing myelopathy (Figure 2).

\section{Figure 2}

Fig. 2 A sagital contrast-enhanced MR image demonstrates diffuse enhancemet of adjacent thoracal vertebral bodies with destruction of the intervertebral disc and vertebral end plates. An enhancing anterior paraspinal soft tissue component and increased cord signal consistent with myelomalacia are also seen (arrows).

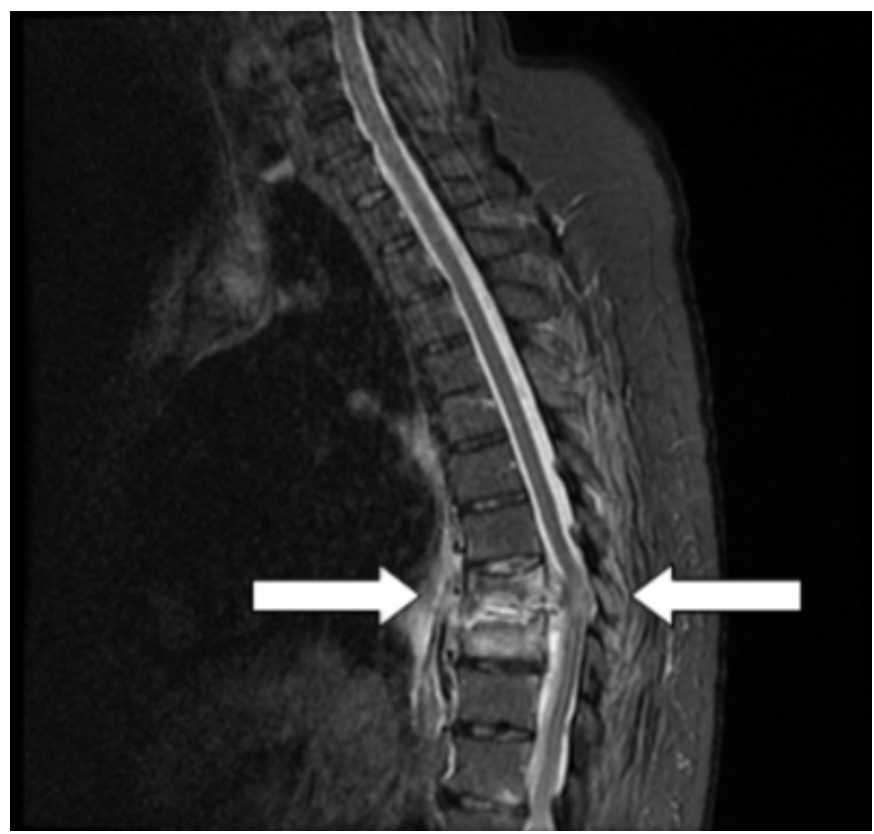

The patient was admitted to hospital for operation after neurosurgery consultation with the diagnosis of posterior epidural abscess. The surgical intervention was accomplished using a posterior approach technigue. Partial laminectomy was done, granulation tissue and yellow purulent collection were drained. Staphylococcus aureus was cultured in the blood culture. A 3-week intravenous therapy followed by 6 weeks of oral antistaphylococcal therapy was instituted. The motor deficit improved from $1 / 5$ to $4 / 5$ in the 4 th week follow-up visit.

\section{DISCUSSION}

Back pain is one of the most challenging diagnosis in EDs because of its rather crowded list of differential diagnosis such as aortic dissection, lumbar disc herniation, nefrolithiasis and spinal epidural masses. Of these, spinal epidural abscess (SEA) is one of the rarest diagnosis. The classical clinical triad, known as fever, spinal pain and neurological deficit, is observed in only a small portion of the cases as seen in our patient. These classical symptoms are non-specific and regrettably in crowded EDs. It is hard to diagnose SEA early because of the nonspecific nature of its symptoms. The lack of related sensitive and specific laboratory tests adds further to the difficulty of early diagnosis. The ratio of correct initial diagnoses of SEA was 
reported to be $11 \%$ in the study of Lu et al. ${ }^{3}$ Most of the clinical findings in SEA overlap with symptoms and findings of other pathological conditions. In nearly $80 \%$ of SEA cases risk factors such as diabetes, cancer, degenerative spinal disease, tumors, AIDS, intravenous drug abuse, alcoholism and steroid intake are present. The most frequently observed risk factor is diabetes mellitus, being present in $18-54 \%$ of cases. Evaluation for risk factors are extremely important with regard to diagnosis. One study suggests that risk factor assessment offers a beter sensitivity than the traditional triad of SEA, without significant loss of specificity. ${ }^{4}$ A detailed history revealed that a deep neck infection as a risc factor for SEA for our patient. Spinal epidural abscess may develop as a spread from a neighboring osteomyelitis focus or as a hematogenous spread from distant sites (usually skin or subcutaneous tissue). ${ }^{4}$ Ventral abscesses are rare and usually the broad dorsal extradural area, which is rich in venous supply, is involved. When a spinal epidural abscess is suspected, leukocyte count, erythrocyte sedimantation rate and Creactive protein may be helpful in showing the infection. The white blood cell count is normal in half of the SEA cases, and if elevation is present, it is usually a moderate one and does not contribute to the SEA diagnosis. Direct roentgenograms provide useful information in only $20 \%$ of cases and it is preferable to order directly MRI for these patients presenting spinal cord compression clinic, because a large number of patients have degenerative changes on direct $\mathrm{X}$-Ray graphies which are confusing and difficult to diagnose. The most effective visualization modality of spinal epidural abscess is MRI. The epidural mass demonstrates homogeneous contrast enhancement in the early course of the disease following intravenous gadolinium administration.

On T1 weighted MR images typical alterations of infected disks have been reported hipointensity and on T2 weighted MR images infected disks are typically considered hyperintense compared with adjacent disks. It is important to realize that disk iso or hypointensity on T2 weighted MR images does not exclude disk infection but may instead represent early infection. ${ }^{5}$

As a conclusion spinal epidural abscess is a rare disease, usually associated with specific predisposing factors; so emergency physician should research risk factors promptly. However, patients may present atypically. Diagnostic delays lead to adverse neurological outcomes. Magnetic resonance imaging is now the diagnostic procedure of choice, if available. Emergency department management should include early administration of dexamethasone and appropriate parenteral antibiotic together with early neurosurgical consultation.

\section{References}

1. Chao D, Nanda A. Spinal epidural abscess: a diagnostic challenge. American Family Physician 2002; 65:1341-46. 2. Soehle M, Wallenfang T. Spinal epidural abscess: clinical manifestations, prognostic factors, and outcomes.

Neurosurgery 2002; 51(1):79-87.

3. Lu CH, Chang WN, Lui CC, et al. Adult spinal epidural abscess: clinical features and prognostic factors. Clinical Neurology and Neurosurgery 2002; 104:306-10.

4. Ergun T, Lakadamyali H, Gokay E. A posterior epidural mass causing paraparesis in a 20 -year-old healthy individual. Internal Journal of Emergency Medicine 2009; 2:195-8. 5. Ledermann HP, Schweitzer ME, Morrison WB, et al. MR Imaging Findings in Spinal Infections: Rules or Myths? Radiology 2003; 228:506-14. 


\section{Author Information}

Pinar Hanife Kara, MD

Emergency Medicine Specialist, Emergency Department, Izmir Ataturk Research and Training Hospital

Ozcan Yavasi, MD

Emergency Medicine Resident, Emergency Department, Izmir Ataturk Research and Training Hospital

\section{Guven Kirimli, MD}

Emergency Medicine Resident, Emergency Department, Izmir Ataturk Research and Training Hospital

\section{Erden Erol Unluer}

Assoc.Prof And Chairman, Emergency Department, Izmir Ataturk Research and Training Hospital

\section{Cengiz Yilmaz, MD}

Radiology Specialist, Radiology Department, Izmir Bozyaka Research and Training Hospital 